\title{
Incidence and patterns of surgical site infections in a teaching hospital in central India
}

\author{
Hedaoo J.B. ${ }^{1}$, Rathod V.N. ${ }^{2}$, Paramne A.V. ${ }^{3}$ \\ ${ }^{1}$ Dr. Jagdish B. Hedaoo, Associate Professor, Department of Surgery, ${ }^{2}$ Dr. Vinod N. Rathod, Associate Professor, \\ Department of Surgery, Shri. Vasantrao Naik Government Medical College, Yavatmal, Maharashtra, India, ${ }^{3}$ Dr. Amit V. \\ Paramne, Senior Resident, Department of Plastic Surgery ${ }^{1,3}$ authors are affiliated with Government Medical College, \\ Nagpur, Maharashtra, India.
}

Corresponding Author: Dr. Vinod N. Rathod, Associate Professor, Department of Surgery, Shri Vasantrao Naik Government Medical College, Yavatmal, Maharashtra, India. E-mail: vinodrathod804@gmail.com

\begin{abstract}
Background: Surgical site infections (SSI) are one of the most common infections among the health care-associated infections. However, there is a scarcity of data on SSI from India. The present study was aimed to determine the incidences of SSI and to evaluate the associated factors at a hospital in Nagpur, central India region. Methods: Surgical sites were considered to be infected according to the set of clinical criteria recommended by the CDC's NNIS system. The wounds were classified using the wound contamination class system, proposed by the American Centers for Disease Control (CDC) for use in SSI surveillance-1999, into Clean, Clean contaminated, Contaminated and Dirty wounds. Results: Among 2083 cases, 314 (15.1\%) were suspected to be clinically infected, among them 250 (12\%) were confirmed to have SSI. Among 2083 cases, 1214 (58.3\%) operations included clean wound category and 869 cases $(41.7 \%)$ were clean contaminated. From clean wounds 58 cases $(4.8 \%)$ were found to be SSI and in clean contaminated wounds $192(22.09 \%)$ cases were found to be SSI. Among the clean wounds, $103(8.5 \%)$ were infected; about 58 cases $(56.3 \%)$ were culture positive and $45(43.7 \%)$ were culture negative. From the clean contaminated wounds, $211(24.3 \%)$, $192(91 \%)$ and $19(9 \%)$ were found to be infected, culture positive and culture negative respectively. The studies of surgeries suggested that among clean surgeries Mastectomy was most common (14\%), whereas Pyelolithotomy (8\%) was the most common among the clean-contaminated surgeries. The prevalence of SSI was highest in Radical cystectomies $(66.6 \%)$ and the least with lipoma excisions (6.4\%). Conclusion: The incidences of SSIs in postsurgical infections were low in addition to this only small numbers of cases were confirmed to be SSIs. Though the reported cases are higher than the countries with high income, a well-planned strategy is required to decrease the incidences of SSI for improving the quality of health care system in present hospital and similar centers elsewhere.
\end{abstract}

Keywords: Surgical Site Infections, Cases, Wounds, Surgery

\section{Introduction}

Though availability of major advancements for controlling infections, the health care induced infections (HII) still remain major problem for public health and their safety globally [1,2]. In studies involving infections globally, it is estimated that occurrence of HII account for approximately 1.4 million cases globally $[3,4]$. Surgical site infections (SSI) are defined infections which occur within thirty days post-surgery or in case of implants the period extends up to one year. These infections can be due to deep incision or may be superficial involving body space or some specific

Manuscript Received: $26^{\text {th }}$ September 2019 Reviewed: $4^{\text {th }}$ October 2019

Author Corrected: $10^{\text {th }}$ October 2019

Accepted for Publication: $16^{\text {th }}$ October 2019 organs [5]. Postoperative surgical site infections are among the most occurring problems in subjects which undergo surgeries and it is the $3^{\text {rd }}$ most frequent identified infection in the population exposed to hospitals [6]. The incidence of SSI varies across various countries and also with the adapted surgical procedures, it is reported that about $2 \%$ of surgeries lead to SSI [7].

In the countries having low to middle income, the incidence of SSI may be at least 5 times higher compared to high-income countries [1]. World health organization (WHO) have suggested that timely surveillance and instructions for surgeons on rate of SSIs along with associated factors can reduce the 


\section{Original Research Article}

occurrence of SSIs up to $50 \%[8,9]$. SSI have serious consequences such as heavy costs for its treatment and also forces long hospital stay. SSI possesses risk of death in patients compared to those subjects who do not developed infection $[10,11]$.

Looking into the serious consequences associated with patients who can develop SSI create a urgent needs for development of strategies for preventing these infections.

In a study performed in United states for the National Healthcare Safety Network which involved survey of approximately of 8.5 lakhs general surgeries, it was evidenced that the overall incidence of SSI was near to $2 \%$ [12], whereas In country like Brazil the incidence of SSI cases for general surgeries ranges from $1.5 \%-40 \%$ [13-14].

It should be noted that, in these studies data involving general surgeries is only presented [13-14]. However, such studies are rare in India reporting the incidence and patterns of SSI.

There are no studies reporting incidence and pattern of SSIs in on such a large patient population.

The study was aimed to demonstrate the incidence rate and the patterns of infections of SSIs in surgical ward of Government Medical hospital in central India, Nagpur.

The outcomes of the present study will provide useful information for surgeons globally working in health care system to decrease the cases and in the associated complications with SSIs.

\section{Methods}

Study setting: The present study was carried out in the Department of Surgery, Government Medical college,
Nagpur, India from June 2009 to November 2011. The surgery ward is a 570 bedded department.

Study participants: All patients admitted between June 2009 and November 2011 was included prospectively in the survey. All nonsurgical cases and patients not undergoing surgery at GMC were not included. Ethical permission was obtained from the Ethics Committee of GMC.

Study design: The present study followed the CDC's NNIS system for inspecting the surgical sites [6]. The classification of wounds was based on wound contamination class system as proposed by the American Centers for Disease Control (CDC) for use in surveillance of SSI, into Clean, Clean contaminated, Contaminated and Dirty wounds.

The surgical sites were inspected at the time of change in dressing after 24 to $48 \mathrm{~h}$ of surgery, at the time swabs were collected from the suspected SSIs and were forwarded for further evaluation followed by postdischarge surveillance if needed.

Swab sampling and laboratory methods: The obtained specimens from swabs were immediately sent to the Department of Pathology, Government Tertiary care hospital, Nagpur, India for further bacteriological study.

All the samples were inoculated onto blood and MacConkey's agar within $2 \mathrm{~h}$ of reception.

The plates were incubated for 24 hour at $37^{\circ} \mathrm{C}$ under aerobic conditions, the plates were observed after 24 hour for any growth.

Statistical analyses: The data was analyzed using GraphPad prism Software for statistics. The mean, standard deviation, range and standard error were evaluated for continuous variables.

\section{Results}

Incidence of surgical site infections: About 2083 cases undergone surgery presented surgical wounds from which 314 cases $(15.1 \%)$ were suspected to be clinically infected. Further it was observed that 250 of the 314 infected wounds studied were found to be culture positive and were considered definite cases of surgical site infection. The overall incidence of infection of 250 cases in 2083 patients was $12 \%$ (Table 1 ).

Table-1: Incidence of SSI.

\begin{tabular}{|l|c|c|}
\hline Wounds studied & $\mathbf{2 0 3 8}$ & -- \\
\hline Clinically Suspected (as per ASEPSIS score) & 314 & $15.1 \%$ \\
\hline No. of Definite SSI (Culture Positive) & 250 & $12 \%$ \\
\hline
\end{tabular}




\section{Original Research Article}

Incidence of surgical site infections according to wound class: In the total of about 2083 patents undergone surgeries, $1214(58.3 \%)$ operations were categorized as Clean wound surgeries, about 58 cases (48\%) were found to be infected.

About 869 cases $(41.7 \%)$ were clean contaminated wounds surgeries.

The incidence of wound infection was significantly high in the clean contaminated wounds, with 192 cases (22.09\%) being infected in 869 surgeries.

A total of 250 cases $(26.89 \%)$ combining clean as well as clean contaminated wound surgeries accounted for SSIs (Table 2).

Table-2: Incidence of SSI according to wound class.

\begin{tabular}{|l|c|c|c|c|}
\hline \multirow{2}{*}{ Wound class } & \multicolumn{2}{|c|}{ Surgeries performed } & \multicolumn{2}{c|}{ SSI } \\
\cline { 2 - 5 } & No. & 58.3 & 58 & 4.8 \\
\hline Clean & 1214 & 41.7 & 192 & 22.09 \\
\hline Clean Contaminated & 869 & $\mathbf{1 0 0}$ & $\mathbf{2 5 0}$ & $\mathbf{2 6 . 8 9}$ \\
\hline Total & $\mathbf{2 0 8 3}$ & & No. & \\
\hline
\end{tabular}

Culture status of SSI isolates: The study of SSI isolates was done for finding the culture positivity of the wounds. From the 103 wounds which were clinically suspected to be infected and studied as the wounds of clean category, 58 (56.3\%) were found to be culture positive.

Among the 211 clinically infected cases of clean contaminated class, 192 cases $(91 \%)$ were found to be culture positive for various organisms.

Among the 314 samples, 64 samples (20.4\%) were found to be culture positive (Table 3 ).

Table-3: Culture positivity in the wounds studied.

\begin{tabular}{|c|c|c|c|c|c|c|c|}
\hline \multicolumn{2}{|l|}{ Wound class } & \multicolumn{2}{|c|}{ Clinically infected } & \multicolumn{2}{|c|}{ Culture positive } & \multicolumn{2}{|c|}{ Culture negative } \\
\hline & NO. & NO. & $\%$ & NO. & $\%$ & NO. & $\%$ \\
\hline Clean & 1214 & 103 & 8.5 & 58 & 56.3 & 45 & 43.7 \\
\hline Clean Contaminated & 869 & 211 & 24.3 & 192 & 91 & 19 & 9 \\
\hline Total & 2083 & 314 & 15.1 & 250 & 79.6 & 64 & 20.4 \\
\hline
\end{tabular}

Rate of infection in various surgeries: From the 2083 cases of enrolled in the study pattern of surgeries done was traced along with the rate of infections which were detected.

Mastectomy was the largest numbered surgical process done numbering 292 cases $(14.01 \%)$ followed by Pyelolithotomy 164 cases (7.87\%), Ureterolithotomy 157 cases (7.53\%) and Thyroidectomy 135 cases (6.48\%) rest of the data can be found in Table 4.

Most of the listed surgeries showed signs of infections suggesting them as SSIs, highest percentage of infections were identified in Radical cystectomies (66.6\%), Esophagectomy (64.7\%), Penectomy (62.5\%) and Abdomino-perineal resection $(53.5 \%)$ while least infected cases were reported in lipoma excisions (6.4\%), Lumpectomy (8\%), Exploratory laprotomy (8.6\%), Cholecystectomy (9.8\%), Wide local excision (9.8\%) and Umbilical hernia repair (11.1\%). 
Original Research Article

Table-4: Infection rate in various surgeries

\begin{tabular}{|c|c|c|c|}
\hline Surgeries & Performed & Infected & Percentage \\
\hline Mastectomy & 292 & 33 & 11.3 \\
\hline Pyelolithotomy & 164 & 21 & 12.8 \\
\hline Prostatectomy & 125 & 20 & 16 \\
\hline Ureterolithotomy & 157 & 17 & 10.8 \\
\hline Thyroidectomy & 135 & 15 & 11 \\
\hline Gastrojejunostomy & 53 & 14 & 26.4 \\
\hline Appendicectomy & 93 & 13 & 14 \\
\hline Subtotal gastrectomy & 40 & 12 & 30 \\
\hline Wide local excision & 122 & 12 & 9.8 \\
\hline Exploratory laprotomy & 116 & 10 & 8.6 \\
\hline Nephrolithotomy & 63 & 10 & 15.8 \\
\hline Cholecystectomy & 102 & 10 & 9.8 \\
\hline Esophagectomy & 17 & 11 & 64.7 \\
\hline Nephrectomy & 49 & 11 & 22.4 \\
\hline Cystolithotomy & 62 & 9 & 14.5 \\
\hline Superficial parotidectomy & 81 & 8 & 9.8 \\
\hline Right hemicolectomy & 23 & 8 & 34.7 \\
\hline Abdomino-perineal resection & 15 & 8 & 53.3 \\
\hline CBD exploration & 35 & 7 & 20 \\
\hline Pyeloplasty & 31 & 6 & 19.3 \\
\hline Liver hydatid cyst excision & 27 & 5 & 18.5 \\
\hline Thoracotomy & 19 & 6 & 31.5 \\
\hline Penectomy & 8 & 5 & 62.5 \\
\hline Lipoma excision & 77 & 5 & 6.4 \\
\hline Incisional hernia repair & 43 & 5 & 11.6 \\
\hline Radical cystectomy & 6 & 4 & 66.6 \\
\hline Adrenalectomy & 12 & 4 & 33.3 \\
\hline Lumpectomy & 25 & 2 & 8 \\
\hline Epigastric hernia repair & 22 & 3 & 13.6 \\
\hline Whipple's surgery & 8 & 3 & 37.5 \\
\hline Umbilical hernia repair & 18 & 2 & 11.1 \\
\hline Splenectomy & 15 & 3 & 20 \\
\hline Sigmoid resection anastomosis & 4 & 2 & 50 \\
\hline Others* & 34 & 10 & 29.4 \\
\hline Total & 2083 & 314 & \\
\hline
\end{tabular}

\section{Discussion}

To our purview this is the first kind of study in India which has involved such a huge number of surgical cases and then investigated the pattern of incidence of $\mathrm{SSI}$ in them. The present study gives an overview in the causative microbes and the types of surgeries involved in post-surgical site infections. The incidence of SSI in the present study ranged near to $12 \%$ which was higher to the studies of SSI reported earlier [7,12-14], the 


\section{Original Research Article}

possible factor contributing for higher SSIs may be attributed to differences in the patient population involved. The present study also evidenced some studies involving SSI which suggested the rate of infection ranging from 4 to $30 \%$ [9]. These high occurrence rates of SSI could be due to deficient infrastructure of the hospital or the center, improperly managed water facilities providing the water to the center, improper or mismanaged human resources such as poorly educated staff without awareness for infections, poor sanitation in the premises and increased patent burden due to large population in addition to this lack of consciousness for infection among the handling staff may also contribute for SSIs [15].

The present study of postoperative wound infections carried out on patients who had undergone surgery in the General surgery Department, about 2083 clean and clean contaminated surgeries were included in the study. Out of these 2083 cases, 314 cases had clinical signs/ symptoms of infection. 250 Surgical Site infections were confirmed by bacteriological study, so the overall infection rate was $12 \%$.

The incidence rate of $12 \%$ SSI in the study is well within the infection rates of $2 \%$ to $30.7 \%$ seen in other studies [16-18]. The difference in incidence rates may be probably due to variations in interpretations of infection or may be due to differences in the population included in the study. Also, a meaningful single infection rate for all types of wounds is not possible, as the likelihood of infection differs in each type of wound.

One of the most known important factors influencing the incidence of postoperative wound infections is wound contamination class. It was evident from the present study that wound contaminated risk class is independently predictive of infection. Among the Clean wounds, which accounted for more than half the number of cases, the rate of infection was only $4.8 \%$.

But in Clean contaminated cases, the rate of infection dramatically increased to $22.09 \%$, probably because of profound influence of endogenous contamination. The incidence rates in the present study are well in accordance with the widely referred incidence rates generated at the Foothills hospital project and other studies $[19,20]$.

The incidence rate in clean wounds in the present study of $4.8 \%$ is slightly higher than those of the other studies in $[19,20]$ and lower than the rates observed by Ojiegbe et al [21].
One of the most important factors which was studied, was the correlation between type of surgeries and rate of infections. It is the study first of its type which established a link relating type of general surgeries and the rate of surgical site infections. From the 2083 cases, Mastectomy emerged as one of the commonest surgical process and which accounted for about 292 cases i.e. $14.01 \%$. Followed by this Pyelolithotomy emerged to be the second highest surgery accounting for 164 cases i.e. $7.87 \%$, Ureterolithotomy was the third highest having 157 cases i.e. $7.53 \%$ and Thyroidectomy was the fourth highest contributing for 135 cases i.e. $6.48 \%$ of the total. Most of the surgeries showed signs of postsurgical infections suggesting them as SSIs.

Though Mastectomy emerged to be one of the commonest surgical processes but showed only 33 infected cases of the total of 292 accounting just $11.3 \%$. Highest percentage of post-surgical infections were identified in Radical Cystectomies i.e. in 4 of the 6 cases $(66.6 \%)$, Esophagectomy in 11 of the 17 cases $(64.7 \%)$, Penectomy in 5 of the 8 cases $(62.5 \%)$ and Abdomino-perineal resection in 8 of the 15 cases $(53.5 \%)$ while least infected cases were reported in lipoma excisions 5 among the total 77 cases (6.4\%), Lumpectomy 2 of 25 cases (8\%), Exploratory laprotomy 10 of 116 cases $(8.6 \%)$, Cholecystectomy 10 of 102 cases $(9.8 \%)$, Wide local excision 12 of 122 $(9.8 \%)$ and Umbilical hernia repair 2 of 18 cases $(11.1 \%)$.

However, more studies verifying types of infection meeting the NHSN guidelines are required for determining the long-term effects of SSI surveillance after the included general and specific surgeries.

More planned and optimized randomized controlled trials should be carried which would benefit the hospitals to follow good practices such as soaking of implants in antibiotics prior to use, more attention to sanitation and imparting education to the staff involved. In addition to this more studies involving standardized infection preventing measures are required to point out specific practices linked with lower risk of SSI in population.

\section{Conclusion}

In conclusion, the present study has given us the knowledge of the incidence of SSI in our hospital and the variation in the incidence rate of infection in clean and clean contaminated wounds. The study has also given us the idea of the relationship between the type of surgeries and the rate of infection. 


\section{Original Research Article}

\section{What the study adds to the existing knowledge?}

In addition to our existing knowledge, the present study provides a correlation between the types of surgeries and the incidence of surgical site infections. This study presents the incidence rate and the patterns of SSIs, it provides a new horizon towards promoting health practices which would be helpful to reduce the cases of post operational infections.

This study will help to decrease incidence of preventable SSI and will not only improve safety but will also lead to quality health care.

\section{Authors contribution}

All the authors contributed equally to the work. Dr. Jagdish B. Hedaoo, Dr. Vinod N. Rathod, Dr. Amit V. Paramne planned the study. Data was collected and processed by Dr. Jagdish B. Hedaoo, Dr. Vinod N. Rathod, Dr. Amit V. Paramne. All the authors read and verified the manuscript.

Conflict of interest: None declared. Funding: Nil, Permission from IRB: Yes

\section{References}

1. World Health Organization. Report on the burden of endemic health care associated infection worldwide: clean care is safer care. Geneva [Switzerland]: WHO Document Production Services; 2011.

2. Pittet D, Allegranzi B, Storr J, Bagheri Nejad S, Dziekan G, Leotsakos A, Donaldson L. Infection control as a major World Health Organization priority for developing countries. J Hosp Infect. 2008; 68(4): 285-292. doi: 10.1016/j.jhin.2007.12.013.

3. World Health Organization. World alliance for patient safety: forward programme 2006-2007. Geneva [Switzerland]: WHO Press; 2006.

4. Hedaoo JB, Rathod VN, Paramne AV. Bacteriology of Surgical Site Infections and Antibiotic Susceptibility Pattern in Isolates of Postoperative Wound Infections. Tehran Uni Med Sci. 2018;5:3-4.

5. Mangram AJ, Horan TC, Pearson ML, Silver LC, Jarvis WR. Guideline for Prevention of Surgical Site Infection, 1999. Centers for Disease Control and Prevention (CDC) Hospital Infection Control Practices Advisory Committee. Am J Infect Control. 1999; 27(2): 97-132. doi: 10.1016/S0196-6553(99)70088-X.
6. de Lissovoy G, Fraeman K, Hutchins V, Murphy D, Song D, Vaughn BB. Surgical site infection: incidence and impact on hospital utilization and treatment costs. Am J Infect Control.2009;37(5):387-397.doi:10. 1016/j. ajic.2008.12.010.

7. WHO. Guideline for safe surgery. 2009. p. 69-80.

8. Ernesto C, Starling F, Horizonte B. Applicability of the national nosocomial infections surveillance system risk index for the prediction of surgical site infections: a review. Braz J Infect Dis. 2007;11(1):134-141. doi: 10. 1590/S1413-86702007000100028

9. Suchitra J, Lakshmidevi N. Surgical site infections: assessing risk factors, outcomes and antimicrobial sensitivity patterns. Afr J Microbiol Res. 2009;3(4): 175-179.

10. Anderson DJ, Podgorny K, Berríos-Torres SI, Bratzler DW, Dellinger EP, Greene L, et al. Strategies to prevent surgical site infections in acute care hospitals. Infect Control Hosp Epidemiol. 2014;35(6): 605-627. doi: 10.1086/676022.

11. Engemann JJ, Carmeli Y, Cosgrove SE, Fowler VG, Bronstein MZ, Trivette SL, et al. Adverse clinical and economic outcomes attributable to methicillin resistance among patients with Staphylococcus aureus surgical site infection. Clin Infect Dis. 2003;36(5):592-598. doi:10. 1086/ 367653.

12. Mu Y, Edwards JR, Horan TC, Berrios-Torres SI, Fridkin SK.Improving risk-adjusted measures of surgical site infection for the national healthcare safety network. Infect Control Hosp Epidemiol. 2011;32(10): 970-986. doi: 10.1086/662016.

13. Agência Nacional de Vigilância Sanitária (ANVISA) Critériosdiagnósticos de infecçãorelacionada à Assistência à Saúde [Internet] Brasília (DF): Ministério da Saúde; 2013.

14.Ercole FF, Chianca TCM, Duarte D, Starling CEF, Carneiro M. Surgical site infection in patients submitted to orthopedic surgery: the NNIS risk index and risk prediction. Rev. Latino- Am. Enfermagem. 2011; 19(2): 269-276. doi:10. 1590/s 0104- 1692011000200 007

15. Mathur P. Hand hygiene: back to the basics of infection control. Indian J Med Res. 2011;134(5):611620. doi: 10.4103/0971-5916.90985. 


\section{Original Research Article}

16. KamatUS, Fereirra AMA, Kulkarni MS, Motghare DD. A prospective study of surgical site infections in a teaching hospital in Goa. Indian J Surg. 2008;70(3): 120-124. doi: 10.1007/s12262-008-0031-y.

17. Nguyen D, MacLeod WB, Phung DC, Cong QT, Nguy $\mathrm{VH}$, Nguyen $\mathrm{H}$, Hamer $\mathrm{DH}$. Incidence and predictors of surgical site infections in Vietnam. Infect Control Hosp Epidemiol. 2001;22(8):485-492. doi:10. 1086/ 501938.

18. Arabshahi K.S., Koohpayezade J. Investigation of risk factors for surgical wound infection among teaching hospitals in Tehran. Int Wound J. 2006;3:5962. doi:10.1111/j.1742-4801.2006. 00176.x.
19. Olson MM, Lee JT. Continuous, 10-year wound infection surveillance. Results, advantages, and unanswered questions. Arch Surg. 1990; 125: 794-803. doi: 10.1001/archsurg.1990.01410180120020.

20. Cruse PJ, Foord R. The epidemiology of wound infection: a 10-year prospective study of 62,939 wounds. Surg Clinic North Am. 1980; 60 (1): 27-40. doi: 10. 1016/s0039-6109(16)42031-1.

21. Ojiegbe GC, Njoku-Obi AN, Ojukwa JO. Incidence and parametric determinants of post-operative wound infections in a university teaching hospital. Cent Afr J Med. 1990; 36 (3):63-67.

\section{How to cite this article?}

Hedaoo J.B, Rathod V.N, Paramne A.V. Incidence and patterns of surgical site infections in a teaching hospital in central India. Surgical Update: Int J surg Orthopedics.2019;5(4):293-299.doi:10.17511/ijoso.2019.i04.10 